\title{
COMBATing Breast Cancer Conference 2011 - Stories of Success and Failure
}

\author{
Julian Puppe ${ }^{a, b} \quad$ Rachel Wuerstlein ${ }^{a, b, c}$ Stefan Kraemer ${ }^{a, b} \quad$ Lisa K. Richters ${ }^{a, b}$ \\ aBreast Center, Department of Obstetrics and Gynecology, University of Cologne, \\ ${ }^{\mathrm{b}} \mathrm{ClO}$ Cologne-Bonn, Cologne, \\ 'Breast Center, LMU Munich, Germany
}

On November 18-19, 2011 the 4th COMBATing (Conference On Molecular Basics $A$ nd Therapeutic Implications In Breast Cancer) meeting took place, for the first time in Munich. This year's meeting had the motto 'Stories of Success and Failure'

The congress, chaired by Nadia Harbeck (Cologne), Manfred Kaufmann (Frankfurt/M.), and Achim Rody (Homburg/ Saar), was an outstanding success. For the more than 250 participants, predominantly young physicians and medical scientists, this conference offered a unique platform for the interdisciplinary exchange and translational networking in breast cancer research. An impressive number of invited national and international speakers gave an overview of recent scientific discoveries in breast cancer research. In 30 lectures they presented the newest developments in basic, translational and clinical research focusing on targeted therapy, predictive markers, subtypes, and tumorous stroma, evaluating their future clinical relevance.

\section{AGO TraFo Meets COMBAT}

For the first time the main conference was preceded by the pre-symposium 'AGO TraFo meets COMBAT' (AGO TraFo = Arbeitsgemeinschaft Gynäkologische Onkologie, Kommission Translationale Forschung), conceived as a platform for young scientists and clinicians interested in translational research. After introducing words by Tanja Fehm (Tübingen), Nadia Harbeck, and Achim Rody the first session was dedicated to young researchers to support their career-planning. Assorted specialists discussed Germany's research landscape. Achim Rody, Peter Fasching (Erlangen), and Brigitte Rack (Munich), shared their own experiences with translational research and showed the importance of interdisciplinary cooperation, networking, and mentoring of young scientists. Annika Thiel from 'Deutsche Krebshilfe e.V.' presented different possibilities of scholarships for young scientists (www.krebsgesellschaft.de).
The second part of the pre-symposium was designed to give the young scientists a platform to present their latest findings in translational research. Out of 34 abstracts submitted by young researchers the best 4 were selected by a scientific committee. The winning abstracts were presented in a special oral session. As winner of the first price, Claus Lattrich (Regensburg), presented his findings entitled 'Estrogen Receptor Agonists Reduce Invasiveness of Triple-Negative Human Breast Cancer Cell Lines' showing the positive influence of estrogen- $\beta$-receptor on patients outcome. During the following lunch break a poster-walk gave the young investigators of the remaining abstracts the chance to present their findings to interested faculty members.

\section{COMBATing 2011}

Immediately after the pre-symposium Nadia Harbeck, Achim Rody, and Manfred Kaufmann heartily welcomed all participants to this year's meeting. After his greeting to the 'young researching elite of our specific field' Klaus Friese (President of DGGG, Munich) expressed his excitement that the number of applications for grants for gynecological research projects doubled this year, representing a growing scientific community in Germany in this field. Anton Scharl (Amberg) from the AGO, and Ingo Bauerfeind (Landshut, TZM) gave an overview on the development of breast cancer research and emphasized the importance of interdisciplinary and translational networking. Regrettably, we had to learn that this conference will be the last headed by Manfred Kaufmann who resigned after chairing 4 years of successful COMBATing meetings.

The first session focused on the new developments since COMBAT 2010. Valuable clues to the substance group that might be the closest associated with the title 'stories of failure' were offered: the PARP-inhibitors. Even though the agent iniparib did not show the expected results in the phase III trial

\section{KARGER}

Fax +497614520714

Information@Karger.de

www.karger.com 
presented at ASCO 2011 by O'Shaughnessy et al., there are many other promising approaches, showing that PARP inhibition is not to be discounted yet (especially considering the fact that iniparib is not a parp-inhibitor in the first place).

In the following session the question of clinical relevance of new targets like PI3K and MEK inhibitors, sphingolipids, regulatory $\mathrm{T}$ cells, and vitamine $\mathrm{D}$ was posed. The tumorous stroma as therapeutic target was in the spotlight of the next session. As first lecturer Thomas Karn (Frankfurt/M.), the recipient of this year's Henner Graeff COMBAT Award, showed in his presentation the importance of the knowledge about the heterogeneity of breast cancer. For the development of better suitable therapies the established differentiation in molecular subtypes is not enough, the patient's microenvironment, especially the lymphocytic reaction, is an important predictive marker in subtype specific analysis. This session clarified in an incomparable way that it is not the cancer we treat, but the patient.

The following session ventured a glimpse into the future. The big challenge for statisticians dealing with the enormous amount of data arising from global molecular networks was presented including proposals for sufficient solutions. Next generation sequencing and its possible clinical implications and targets for the future were shown.

Friday's talks were finalized by the key note lecture held by Peter Schmid (Brighton), entitled 'DNA-Repair: Therapeutic Applications for Today and Tomorrow'. He gave a comprehensive overview of the DNA-repair mechanisms and their clinical relevance especially for triple-negative breast cancer (TNBC), even though many pathways are not known yet. He described the principle of synthetic lethality, which was used to develop the PARP inhibitors. Asking the question why not all patients profit from such targeted therapy he demonstrated the enormous heterogeneity of breast cancer; even in TNBC at least 7 different biological clusters exist. Gaining better insights into DNA repair mechanisms may lead to new targeted approaches.

The first day was completed by a social evening. During this get-together 10 travel grants were awarded by the scientific chairs. They were given to young physicians and scientists who showed special interest in and commitment to breast cancer research. This year the COMBAT Award was renamed 'Henner Graeff COMBAT Award' in honor of Henner Graeff, the deceased former head of the gynecological department at TU Munich, who dedicated his life to gynecological research, being the first translational researcher in Germany in this field. Manfred Schmitt (Munich) delivered a personal honorific speech in attendance of Mrs. Graeff. The award was given to Thomas Karn (fig. 1), who was the first basic researcher who received this honor; his predecessors were gynecologists.

The next day started with a keynote lecture by Lajos Pusztai (Houston, TX) evaluating the current role of prognostic signatures in breast cancer beyond molecular classification

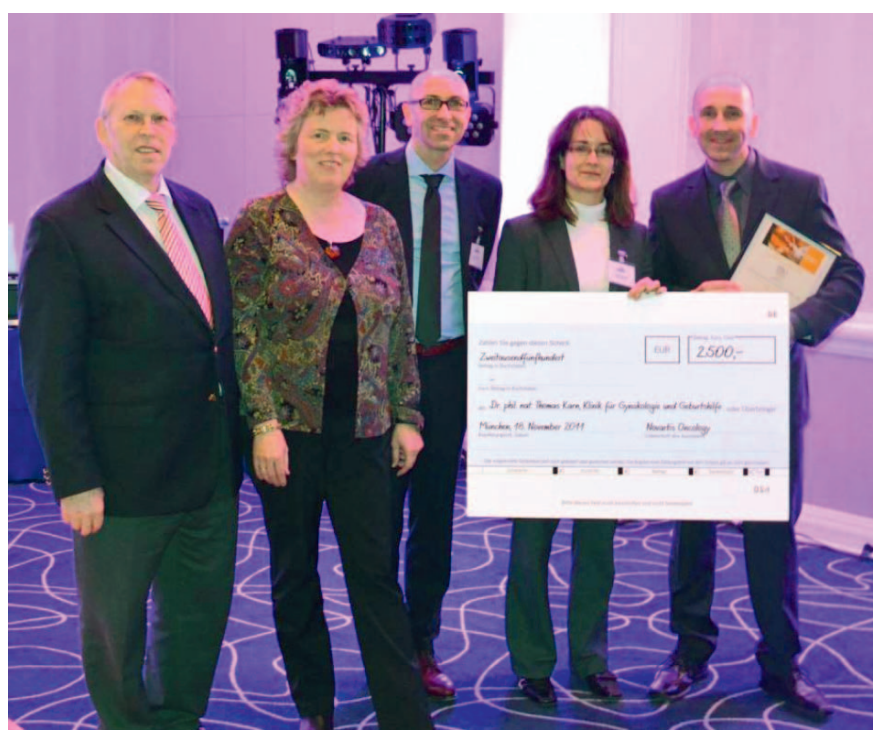

Fig. 1. The scientific chairs Manfred Kaufmann, Nadia Harbeck, and Achim Rody presenting the first Henner Graeff COMBAT Award to Thomas Karn. The award was sponsored by Novartis Oncology (represented by Mrs. Schmidt).

and gene expression. In the last years molecular profiling was successfully implemented in clinical routine to identify patients who obtain the most therapeutic benefit from chemotherapy, e.g. by using Oncotype $\mathrm{DX}^{\circledR}$ or Mammaprint ${ }^{\circledR}$. But many clinically important prediction problems, such as in estrogen receptor negative (ER-) cancers are difficult to address, with currently available data demonstrating the limits of these new techniques. 'Since each cancer harbors a unique combination of mutations, DNA copy number aberrations, and mRNA expression anomalies, we should use the genomic data to generate clinically testable therapeutic hypotheses and identify the next generation of drug targets for patient tailored treatments. But with too many therapeutic biomarkers to test we face a new challenge' concluded Lajos Pusztai. New trials might help to evaluate novel patient oriented therapies by combining molecular analysis with a portfolio of markers directed therapeutic phase II trials.

In the same context the next session discussed molecular subtypes with their clinical consequences. Unfortunately, we still have no directed therapy for TNBC. Data of the ABCSG on prediction of endocrine response showed that a high Ki67 proliferation index is associated with poor outcome in postmenopausal ER+ breast cancer. In addition, new data about ErbB (HER) receptor subtypes demonstrated that overexpression of the HER4-receptor might play an important role in targeted therapy because of its positive influence on patient's outcome when combined with ER positivity.

In the next session the pharmaceutical industry was given a platform to present recent developments in tailored breast cancer therapy. Roche gave an update on antibody drug conjugates with T-DM1 and dual HER2/neu inhibition with pertuzumab by introducing the APHINITY trial. By presenting 
data of the BOLERO trials with the mTOR everolismus (RAD-001), Novartis showed a modern way of individualized therapy in breast cancer. Furthermore, a change in clinical trial design to more efficient and smaller studies by selecting patients with distinct gene mutations for targeted small molecules was discussed, anticipating higher response rates and faster results.

The session on troubleshooting in translational research discussed the problems and solutions in clinical study development and realizing of big study groups like the WSG and ABCSG in comparison to other networks. Hans Tesch (Frankfurt/M.) stressed the importance of health service research for breast cancer therapy. He showed that clinical trials especially for rare tumor entities could also be successfully conducted in a network of oncology practices in collaboration with local breast centers.

During the session on challenging histological subtypes, it was shown that the medullary subtype has a good prognosis and may not require any adjuvant chemotherapy if node-negative. Also the molecular characteristics of inflammatory and metaplastic breast carcinoma were part of this session. It was shown that the majority of male breast cancers belong to the luminal $\mathrm{A} / \mathrm{B}$ subtype and a new clinical study for this rare male tumor was presented including a translational research program.

With regard to the motto of this meeting 'Stories of Success and Failure' the last session was designated to evaluation of the 'old targets' in targeted breast cancer therapy starting with a critical overview of the increasing number of biomarkers published in the last years and focusing on the rediscovery of cancer stem cells as useful target for breast cancer treatment. As one representative success story the implementation of endocrine therapy for breast cancer with the 'old and successful drug' tamoxifen and aromatase-inhibitors (AIs) was discussed. Subsequently, the inhibition of the mTOR pathway was highlighted and recent phase II and III studies demonstrated a high efficacy of AIs and tamoxifen combined with mTOR inhibition. Also mTOR inhibition seems to have the potential to overcome HER2/neu and endocrine resistance and might sensitize TNBC to platinum drugs. Unfortunately, sufficient data on mTOR signaling in HER2/neu and TNBC is still lacking. Nevertheless, mTOR inhibition is a success story in breast cancer treatment with a high potential for clinical routine.
As an example for a failed treatment regimen, anti-angiogenetic therapy with the VEGF-inhibitor bevacizumab was presented. Recent confirmatory studies showed no quality of life or survival advantage for bevacizumab-containing regimens in metastatatic breast cancer and further studies are needed to identify the right patients for anti-angiogenetic therapies.

The conference was closed with news on the application of tyrosine kinase inhibitors in breast cancer demonstrating that combined HER2/neu inhibition with trastuzumab and lapatinib has superior anti tumor activity in neoadjuvant therapy and metastatic breast cancer. Taken together, dual HER2/neu inhibition is another success in breast cancer treatment and further studies should investigate the synergism of mTOR inhibitors with HER2/neu-inhibition.

In summary, the organizers of the COMBATing conference provided an excellent meeting on translational research by inviting the German leading experts in the field of breast cancer as well as international keynote speakers. With selected talks about the promising achievements on the one hand and the disappointing results on the other hand, the conference reflected the current problems of breast cancer treatment. It was pointed out that the perspective of breast cancer research will be to specify the different subtypes in genetic, molecular, and biological ways without disregarding the tumor's surrounding to select the right targeted therapy for the right patient.

Furthermore, the first joining of the TRAFO meeting with the COMBATing conference served as an ideal platform for social and career networking and was an exciting teaching lesson especially for young gynecologists and scientists interested in translational research. This fruitful combination should be continued in the coming years.

The meeting also stressed the importance of interdisciplinary exchange between basic researchers, clinicians, study groups, and also the pharmaceutical industry to speed up the implementation of new targets and treatment strategies into the clinical setting. This unique mélange makes this conference the most important in translational breast cancer research in Germany. The next COMBATing Breast Cancer Conference will be held in Munich, November 23-24, 2012. More information about this conference at www.combating.de. 